\title{
SUPPLEMENTARY MATERIALS: THE SCREENING AND RANKING ALGORITHM FOR CHANGE-POINTS DETECTION IN MULTIPLE SAMPLES
}

\author{
CHI SONG, XIAOYI MIN, AND HEPING ZHANG
}

\section{Simulation of DATA With COMMON AND INDividual TREND}

We evaluate how the change-point detection could be affected by underlying trends that are affecting all the samples but slightly different in each individual. To this end, we simulated data by introducing a systematic trend. Otherwise, the rest of the simulation procedure was the same as that in Section 4.2.1. Specifically, we simulated $\mathbf{Y}_{i}$ by adding both random noise and trend as follows

$$
Y_{i, t}=\mu_{i, t}+0.12 \sin (2 \pi t / 96+\psi)+0.24 \sin \left(2 \pi t / 240+\phi_{i}\right)+\epsilon_{i, t},
$$

where $\psi \sim U(0,2 \pi), \phi_{i} \sim U(0,2 \pi)$, and $\epsilon_{i, t} \sim N(0,1)$. In this model, the term $0.12 \sin (2 \pi t / 96+$ $\psi)$ is the trend shared by all samples, and $0.24 \sin \left(2 \pi t / 240+\phi_{i}\right)$ is the trend unique for each sample. Five representative sequences are given in Figure 1. Similar to Table 1, Supplement Table 1 presents the number of shared change-points detected. We can see that the trends exercised great impact on the single sample methods. Multiple-sample CBS also yielded a notable number of false change-points. In fact, we considered various scenarios with different period and magnitude of the trends. Not surprisingly, the performance of multiple-sample CBS, and of course the single sample methods, became worse. Fortunately, our multiple-sample m-SaRa procedure performed robustly, detecting all 6 true change-points in 996 out of 1000 replicates.

\section{DAta With DEPEndent ERRors}

Even though our method and most other methods assume independent errors, we would like to simulate situations with correlated errors and find out how robust the methods are when the independent error assumption is violated.

To evaluate the effect of dependent errors, we simulated data using the same mean model as specified in Section 4.2.1. Instead of assuming $\boldsymbol{\epsilon}_{i} \sim \operatorname{MVN}(0, \mathbf{I})$, we simulated $\boldsymbol{\epsilon}_{i}$ from a moving average model of order 20, with all the 20 parameters equal 0.01 . Similarly, Table 2 shows the number of shared change-points detected. Compared with Table 1, we notice that the correlated errors increased the false positive in single sample methods but did not affect the performance of the multiple sample methods. An explanation similar to Section 1 applies here, because the effects of correlated errors on each sample is similar to random short trends. 


\section{Proof of TheOrem 1}

Let $h=L / 2$, then with arguments similar to Lemma 3 in Niu and Zhang $(2012)$, for any combining statistic $W(t, h)$, it suffices to show that there exists $\lambda$ such that as $N \rightarrow \infty$,

$$
\begin{aligned}
& P(W(t, h)<\lambda) \rightarrow 1, \text { for any } h \text {-flat point } t, \\
& P\left(W\left(\tau_{j}, h\right)<\lambda\right) \rightarrow 0, \text { for any } j \in\{1, \ldots, J\} .
\end{aligned}
$$

We first prove (1) and (2) for $W^{\text {Sum }}, W^{\text {WSum }}, W^{\text {Fisher }}$, and $W^{\text {Stouffer }}$ by proving them for a general class of combining statistics of the following form

$$
W(t, h)=\frac{1}{N} \sum_{i=1}^{N} f\left[\tilde{D}_{i}(t, h)\right]
$$

where $f(\cdot)$ is a function satisfying conditions $(\mathrm{C} 1)$ and $(\mathrm{C} 2)$ specified below.

Let $Z_{0} \sim N(0,1)$ and $Z_{j}$ follows the same distribution as $\tilde{D}_{i}\left(\tau_{j}, h\right)($ for $j=1, \ldots, J)$.

(C1) $E\left|f\left(Z_{0}\right)\right|^{3}$ and $E\left|f\left(Z_{j}\right)\right|^{3}(j=1, \ldots, J)$ exist.

$(\mathrm{C} 2) \nu_{0} \equiv \operatorname{Ef}\left(Z_{0}\right)<\nu_{j} \equiv \operatorname{Ef}\left(Z_{j}\right)$ for any $j$.

It can be shown that $W^{\text {Sum }}, W^{\text {WSum }}, W^{\text {Fisher }}$, and $W^{\text {Stouffer }}$ all follow (3) and satisfy (C1). They also satisfy (C2) when $\eta_{j}^{2}>1$ or when $\eta_{j}^{2}=1$ and $\Delta_{j} \neq 0$.

Next, we show that (1) and (2) hold for (3) with the two conditions specified above. We let $\zeta=\min _{j}\left(\nu_{j}-\nu_{0}\right) / 2, \lambda=\nu_{0}+\zeta$, and $\zeta_{j}=\zeta / \sqrt{\operatorname{Var}\left[f\left(Z_{j}\right)\right]}$ for $j=0, \ldots, J$. For (1), note that for an $h$-flat point $t$,

$$
P(W(t, h)<\lambda)=P\left(\frac{W(t, h)-\nu_{0}}{\sqrt{\operatorname{Var}\left[f\left(Z_{0}\right)\right] / N}}<\sqrt{N} \zeta_{0}\right)
$$

which can be approximated by the corresponding normal probability with the difference being controlled by the non-uniform Berry-Esseen bound (see e.g. Theorem 14 on Page 125 of Petrov (1975)) as follows.

$$
\begin{aligned}
& P(W(t, h)<\lambda) \\
\geq & \Phi\left(\sqrt{N} \zeta_{0}\right)-\frac{C \cdot E\left|f\left(Z_{0}\right)-\nu_{0}\right|^{3}}{\sqrt{N}\left\{\operatorname{Var}\left[f\left(Z_{0}\right)\right]\right\}^{3 / 2}\left(1+\sqrt{N} \zeta_{0}\right)^{3}} \\
\geq & 1-\frac{1}{\sqrt{2 N \pi} \zeta_{0}} \exp \left(-\frac{1}{2} N \zeta_{0}^{2}\right)-\frac{C \cdot E\left|f\left(Z_{0}\right)-\nu_{0}\right|^{3}}{N^{2} \zeta^{3}} \\
\rightarrow & 1, \text { as } N \rightarrow \infty,
\end{aligned}
$$

where $C$ is an absolute constant. 
Similarly, since $\nu_{j}>\lambda$,

$$
\begin{aligned}
& P\left(W\left(\tau_{j}, h\right)<\lambda\right) \\
\leq & \Phi\left(-\sqrt{N} \zeta_{j}\right)+\frac{C \cdot E\left|f\left(Z_{j}\right)-\nu_{j}\right|^{3}}{\sqrt{N}\left\{\operatorname{Var}\left[f\left(Z_{j}\right)\right]\right\}^{3 / 2}\left(1+\sqrt{N} \zeta_{j}\right)^{3}} \\
\leq & \frac{1}{\sqrt{2 N \pi} \zeta_{j}} \exp \left(-\frac{1}{2} N \zeta_{j}^{2}\right)+\frac{C \cdot E\left|f\left(Z_{j}\right)-\nu_{j}\right|^{3}}{N^{2} \zeta^{3}} \\
\rightarrow & 0, \text { as } N \rightarrow \infty .
\end{aligned}
$$

Therefore, we prove the sure coverage property for $W^{\text {Sum }}, W^{W \text { Sum }}, W^{\text {Fisher }}$, and $W^{\text {Stouffer }}$.

For $W^{H C}$, the sure coverage property follows directly from Theorem 7 in Cai et al. (2011) which showed that, in our setting, letting $\lambda=\sqrt{2(1+\rho) \log \log N}$ for a positive number $\rho$ guarantees (1) and (2).

For adaptive Fisher's method, we consider $f^{\text {Fisher }}(\cdot)=-\log \{2[1-\Phi(|\cdot|)]\}$. We verified $(\mathrm{C} 1)$ and $(\mathrm{C} 2)$ for $f^{\text {Fisher }}$ before and continue to use the notation $\nu_{j}(j=0 \ldots, J)$ and $\zeta$. Recall that for a flat-point $t, W^{A F}(t, h)=\max _{1 \leq i \leq N}\left|\tilde{V}_{i}(t, h)\right|$ where for each $1 \leq i \leq N$, $\tilde{V}_{i}(t, h)$ is a standardized sum of independent exponential random variables. In this case, let $\lambda=\sqrt{N} \zeta$ and we have

$$
\begin{aligned}
P\left(W^{A F}(t, h)<\sqrt{N} \zeta\right) & =P\left(\bigcap_{i=1}^{N}\left\{-\sqrt{N} \zeta<\tilde{V}_{i}(t, h)<\sqrt{N} \zeta\right\}\right) \\
& \geq 1-\sum_{i=1}^{N}\left[1-P\left(-\sqrt{N} \zeta<\tilde{V}_{i}(t, h)<\sqrt{N} \zeta\right)\right],
\end{aligned}
$$

where according to Result 23 on Page 132 of Petrov (1975), for an absolute constant $C$,

$$
\begin{aligned}
& P\left(-\sqrt{N} \zeta<\tilde{V}_{i}(t, h)<\sqrt{N} \zeta\right) \\
\geq & 1-\sqrt{\frac{2}{N \pi \zeta^{2}}} \exp \left(-\frac{1}{2} N \zeta^{2}\right)-\frac{2 C}{(1+\sqrt{N} \zeta)^{3}} \cdot \frac{\sum_{k=1}^{N} w^{3}(k, i)}{\left[\sum_{k=1}^{N} w^{2}(k, i)\right]^{3 / 2}} \\
\geq & 1-\sqrt{\frac{2}{N \pi \zeta^{2}}} \exp \left(-\frac{1}{2} N \zeta^{2}\right)-\frac{2 C}{(1+\sqrt{N} \zeta)^{3}} .
\end{aligned}
$$

Therefore,

$$
\begin{aligned}
& P\left(W^{A F}(t, h)<\sqrt{N} \zeta\right) \\
\geq & 1-\sqrt{\frac{2 N}{\pi \zeta^{2}}} \exp \left(-\frac{1}{2} N \zeta^{2}\right)-\frac{2 N C}{(1+\sqrt{N} \zeta)^{3}} \\
\rightarrow & 1, \text { as } N \rightarrow \infty .
\end{aligned}
$$


On the other hand, for each $j \in\{1, \ldots, J\}$,

$$
\begin{aligned}
& P\left(W^{A F}\left(\tau_{j}, h\right)>\sqrt{N} \zeta\right) \\
\geq & P\left(\left|\tilde{V}_{N}\left(\tau_{j}, h\right)\right|>\sqrt{N} \zeta\right) \\
= & 1-P\left(-\sqrt{N} \zeta<\tilde{V}_{N}\left(\tau_{j}, h\right)<\sqrt{N} \zeta\right) \\
= & 1-P\left(-\zeta<\frac{1}{N} \sum_{i=1}^{N} f^{\text {Fisher }}\left[\tilde{D}_{i}\left(\tau_{j}, h\right)\right]-\nu_{0}<\zeta\right) \\
\rightarrow & 1, \text { as } N \rightarrow \infty .
\end{aligned}
$$

This concludes our proof of the sure coverage property for the adaptive Fisher's method.

\section{Proof of Theorem 2}

For Part (a), we can show that $W^{\text {Sum }}, W^{\text {WSum }}, W^{\text {Fisher }}$, and $W^{\text {Stouffer }}$ all follow (3) and satisfy Condition (C3) specified below.

Let $Z_{0} \sim N(0,1)$ and $Z_{j}$ follows the same distribution as $\tilde{D}_{i}\left(\tau_{j}, h\right)($ for $j=1, \ldots, J)$.

(C3) The moment generating function of the random variable $f\left(Z_{j}\right)$, namely $M_{j}(\beta) \equiv$ $E\left\{\exp \left[\beta f\left(Z_{j}\right)\right]\right\}$ exists on a neighborhood $B_{0}$ of 0 for $j=0,1, \ldots, J$.

Note that $(\mathrm{C} 1)$ and $(\mathrm{C} 2)$ still holds for these combining methods. We can define $\lambda$ respectively for each combining method as in Theorem 1 that satisfies (1). This threshold is more conservative than what is required by the current theorem, so it suffices to show that with this threshold, (2) holds with a rate of convergence exponential in $N$.

Under Condition (C3), Cramer-Chernoff Theorem gives

$$
\lim _{N \rightarrow \infty} \frac{1}{N} \log P\left(W\left(\tau_{j}, h\right)<\lambda\right)=-I_{j}(\lambda),
$$

where $I_{j}(x)=\sup _{\beta \in B_{0}}\left[\beta x-\log M_{j}(\beta)\right]$. Since $\lambda \neq \nu_{j}, I_{j}(\lambda)>0$. Therefore, the claim is true for $W^{\text {Sum }}, W^{\text {WSum }}, W^{\text {Fisher }}$, and $W^{\text {Stouffer }}$.

To prove the claim for the higher criticism method, note that for each $j$, we can find $0<x_{j}<1$ such that $P\left(p_{i}\left(\tau_{j}, h\right)<x_{j}\right) \neq x_{j}$. With arguments similar to those in Cai et al. $(2011)$

$$
\begin{aligned}
& P\left(W^{H C}\left(\tau_{j}, h\right) \leq \sqrt{2(1+\rho) \log \log N}\right) \\
= & P\left(\sup _{0<x<1} \sqrt{N}\left|\frac{\frac{1}{N} \sum_{i=1}^{N} \mathbf{1}_{\left\{p_{i}\left(\tau_{j}, h\right)<x\right\}}-x}{\sqrt{x(1-x)}}\right| \leq \sqrt{2(1+\rho) \log \log N}\right) \\
\leq & P\left(\left|\frac{1}{N} \sum_{i=1}^{N} \mathbf{1}_{\left\{p_{i}\left(\tau_{j}, h\right)<x_{j}\right\}}-x_{j}\right| \leq \sqrt{\frac{2 x_{j}\left(1-x_{j}\right)(1+\rho) \log \log N}{N}}\right) .
\end{aligned}
$$

Since $E\left(\mathbf{1}_{\left\{p_{i}\left(\tau_{j}, h\right)<x_{j}\right\}}\right)=P\left(p_{i}\left(\tau_{j}, h\right)<x_{j}\right) \neq x_{j}$, we can apply the Cramer-Chernoff Theorem again and obtain an upper bound converging to zero exponentially fast as in (4). 
For the adaptive Fisher's method, with the same notation as Theorem 1,

$$
P\left(W^{A F}\left(\tau_{j}, h\right)<\sqrt{N} \zeta\right) \leq P\left(\nu_{0}-\zeta<\frac{1}{N} \sum_{i=1}^{N} f^{\text {Fisher }}\left[\tilde{D}_{i}\left(\tau_{j}, h\right)\right]<\nu_{0}+\zeta\right) .
$$

Then since $E\left\{f^{\text {Fisher }}\left[\tilde{D}_{i}\left(\tau_{j}, h\right)\right]\right\}>\nu_{0}+\zeta$, we can again show it converges to zero at a rate at least exponential in $N$.

For Part (b), we use notation $A_{N} \sim B_{N}$ to denote that $\lim _{N \rightarrow \infty} A_{N} / B_{N}=1$ for two sequences $\left\{A_{N}\right\}$ and $\left\{B_{N}\right\}$. We first find the order of $\lambda^{*}$. For an $h$-flat point $t$, we have

$$
P\left(\max _{i}\left|\tilde{D}_{i}(t, h)\right|>\lambda^{*}\right)=1-\left\{1-2\left[1-\Phi\left(\lambda^{*}\right)\right]\right\}^{N}=\alpha .
$$

This suggests that $\lim _{N \rightarrow \infty} \lambda^{*}=\infty$ and thus by Mill's ratio $1-\Phi\left(\lambda^{*}\right) \sim \frac{1}{\lambda^{*}} \phi\left(\lambda^{*}\right)$. With simple calculation, the formula above leads to $\frac{1}{\lambda^{*}} \phi\left(\lambda^{*}\right) \sim-\log (1-\alpha) / 2 N$. Solve for $\lambda^{*}$, we can show that $\lambda^{*} \sim \sqrt{2 \log N}$, or more precisely,

$$
\lim _{N \rightarrow \infty}\left(\lambda^{*}\right)^{2}-2 \log N+\log \log N+\log \left[\pi \log ^{2}(1-\alpha)\right]=0
$$

Second, we find the asymptotic power of detecting change-point $\tau_{j}$, which is

$$
\begin{aligned}
& P\left(\max _{i}\left|\tilde{D}_{i}\left(\tau_{j}, h\right)\right|>\lambda^{*}\right) \\
& \quad=1-\left\{1-2\left(1-p_{j}\right)\left[1-\Phi\left(\lambda^{*}\right)\right]-p_{j}\left[1-\Phi\left(\frac{\lambda^{*}-\psi_{j}}{\eta_{j}}\right)-\Phi\left(\frac{-\lambda^{*}-\psi_{j}}{\eta_{j}}\right)\right]\right\}^{N} \\
& \quad \equiv 1-\left(1-P_{1}-P_{2}\right)^{N}
\end{aligned}
$$

where $\psi_{j}=-\Delta_{j} \sqrt{h / 2}$. Note that

$$
P_{2} \sim \frac{C}{\lambda^{*}} \exp \left[\frac{-\left(\lambda^{*}\right)^{2}+2\left|\psi_{j}\right| \lambda^{*}}{2 \eta_{j}^{2}}\right],
$$

where $C$ denotes a generic constant. Since $\eta_{j}^{2} \geq 1, P_{2} / P_{1} \rightarrow \infty$, as $N \rightarrow \infty$,

$$
\begin{aligned}
& \log \left[1-P\left(\max _{i}\left|\tilde{D}_{i}\left(\tau_{j}, h\right)\right|>\lambda^{*}\right)\right] \\
\sim & -N \frac{C}{\lambda^{*}} \exp \left[\frac{-\left(\lambda^{*}\right)^{2}+2\left|\psi_{j}\right| \lambda^{*}}{2 \eta_{j}^{2}}\right] \\
\sim & -C \cdot N^{1-\frac{1}{\eta_{j}^{2}}}(\log N)^{-\frac{1}{2}+\frac{1}{2 \eta_{j}^{2}}} \exp \left(\frac{\left|\psi_{j}\right|}{\eta_{j}^{2}} \sqrt{2 \log N}\right),
\end{aligned}
$$

which tend to $-\infty$ as $N \rightarrow \infty$ but slower than $-N$. Thus, the theorem is proved.

\section{REFERENCES}

Cai, T. T., Jeng, X. J., and Jin, J. (2011). Optimal detection of heterogeneous and heteroscedastic mixtures. Journal of the Royal Statistical Society: Series B, 73(5), 629662. 
Niu, Y. S. and Zhang, H. (2012). The screening and ranking algorithm to detect DNA copy number variations. The Annals of Applied Statistics, 6(3), 1306-1326.

Petrov, V. (1975). Sums of independent random variables. Ergebnisse der Mathematik und ihrer Grenzgebiete. Springer-Verlag. 


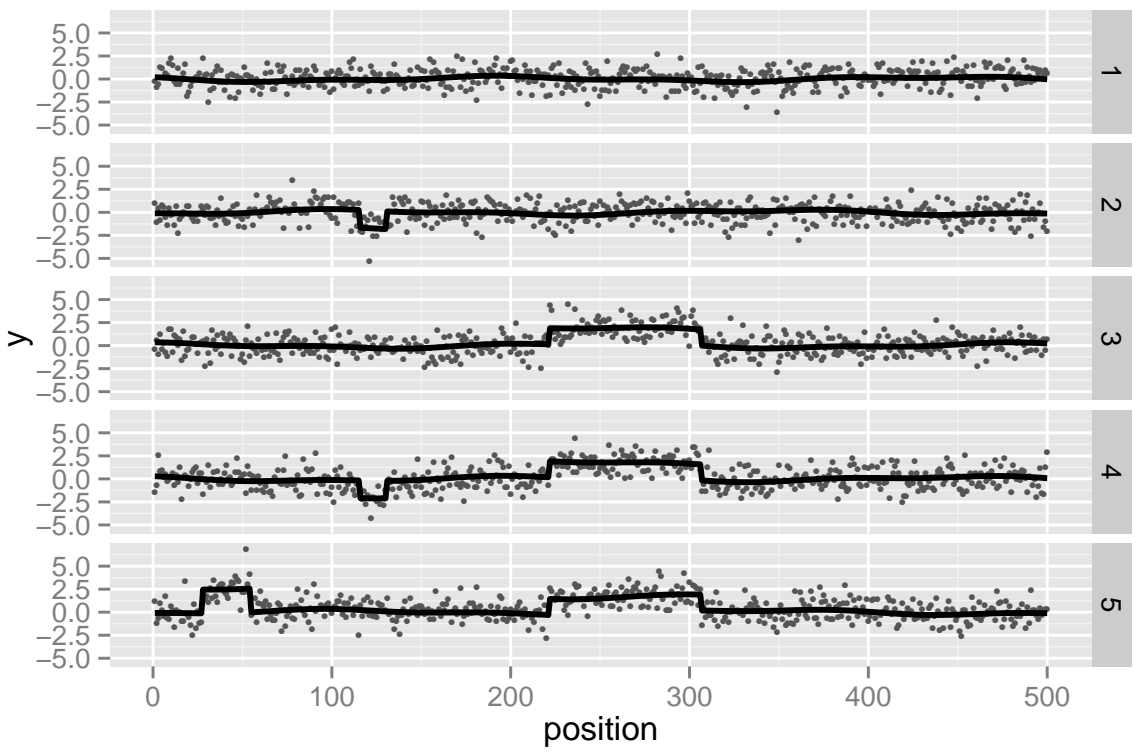

Supplement Figure 1. The simulated data with trend. Five samples are shown. The mean signals without noise are shown by bold black lines. 


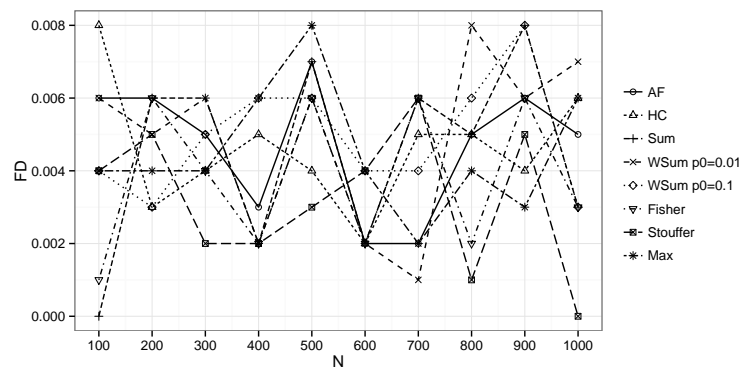

(a) Average number of false discoveries for the rare change-point simulated in Section 4.1

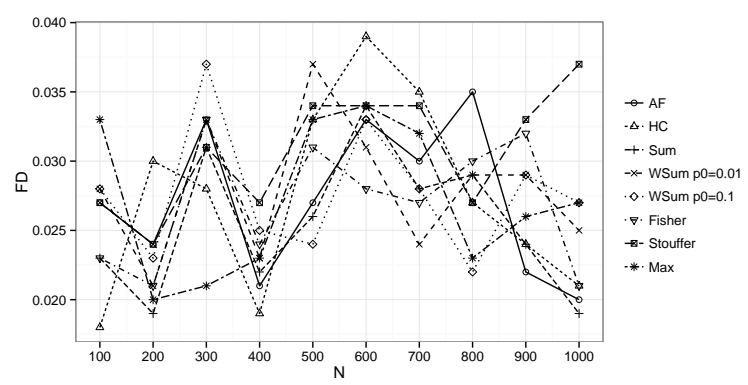

(b) Average number of false discoveries for the common change-point simulated in Section 4.1

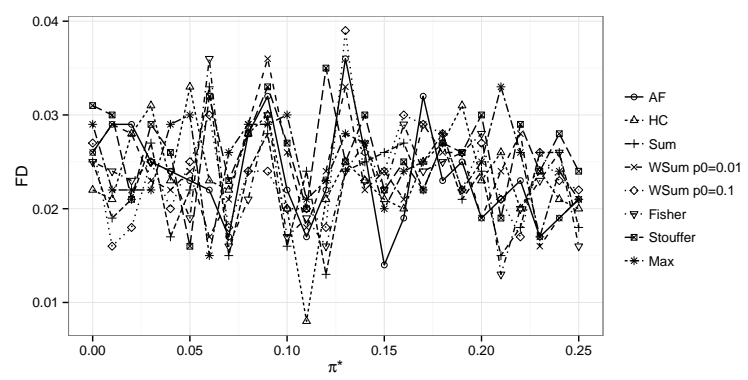

(c) Average number of false discoveries of different methods as $\pi^{*}$ changes in Section 4.1

Supplement Figure 2. Average number of false discoveries for simulations in Section 4.1. 
Supplement TABle 1. The number of shared change-points detected for the simulation with trend.

\begin{tabular}{cccccc}
\hline \hline & \multicolumn{7}{c}{ Number of change-points } \\
\hline Method & $\leq 5$ & $\mathbf{6}$ & 7 & 8 & $>8$ \\
\hline fast CBS & 0 & $\mathbf{0}$ & 0 & 0 & 1000 \\
CBS-SS & 0 & $\mathbf{0}$ & 0 & 0 & 1000 \\
m-SaRa & 0 & $\mathbf{0}$ & 0 & 0 & 1000 \\
Multiple-sample CBS & 3 & $\mathbf{7 4 9}$ & 231 & 8 & 9 \\
Multiple-sample m-SaRa & 0 & $\mathbf{9 9 6}$ & 4 & 0 & 0 \\
\hline
\end{tabular}


Supplement TABLE 2. The number of shared change-points detected for the simulation with dependent errors.

\begin{tabular}{cccccc}
\hline \hline & \multicolumn{7}{c}{ Number of change-points } \\
\hline Method & $\leq 5$ & $\mathbf{6}$ & 7 & 8 & $>8$ \\
\hline fast CBS & 0 & $\mathbf{3 1}$ & 93 & 229 & 647 \\
CBS-SS & 0 & $\mathbf{1 3 7}$ & 312 & 314 & 237 \\
m-SaRa & 0 & $\mathbf{0}$ & 0 & 0 & 1000 \\
Multiple-sample CBS & 0 & $\mathbf{1 0 0 0}$ & 0 & 0 & 0 \\
Multiple-sample m-SaRa & 0 & $\mathbf{1 0 0 0}$ & 0 & 0 & 0 \\
\hline
\end{tabular}


Supplement Table 3. True and false positives grouped by the changepoints (CP1-CP6) for the simulation with trend in Supplement Section 1. Standard errors are shown in parentheses.

(a) Average number of true positives.

\begin{tabular}{ccccccc}
\hline \hline & CP1 & CP2 & CP3 & CP4 & CP5 & CP6 \\
Number of Carriers & 20 & 20 & 50 & 50 & 100 & 100 \\
\hline fast CBS & $19.8(0.4)$ & $19.8(0.4)$ & $46.4(1.9)$ & $46.4(2.0)$ & $92.1(2.7)$ & $92.2(2.8)$ \\
CBS-SS & $19.8(0.4)$ & $19.8(0.4)$ & $46.3(1.9)$ & $46.3(2.0)$ & $91.9(2.7)$ & $92.1(2.8)$ \\
m-SaRa & $19.8(0.5)$ & $19.8(0.5)$ & $47.0(1.7)$ & $47.1(1.6)$ & $91.4(2.8)$ & $91.3(2.9)$ \\
Multiple-sample CBS & $20.0(0.0)$ & $20.0(0.0)$ & $49.9(0.3)$ & $49.9(0.3)$ & $100.0(0.0)$ & $100.0(0.0)$ \\
Multiple-sample m-SaRa & $20.0(0.0)$ & $20.0(0.0)$ & $49.8(0.5)$ & $49.8(0.5)$ & $100.0(0.0)$ & $100.0(0.0)$ \\
\hline
\end{tabular}

(b) Average number of false positives.

\begin{tabular}{ccccccc}
\hline \hline & CP1 & CP2 & CP3 & CP4 & CP5 & CP6 \\
\hline fast CBS & $6.9(2.8)$ & $8.0(3.0)$ & $7.5(2.9)$ & $7.5(2.8)$ & $6.2(2.6)$ & $6.5(2.7)$ \\
CBS-SS & $1.9(1.4)$ & $4.0(2.1)$ & $5.5(2.5)$ & $5.7(2.5)$ & $5.0(2.3)$ & $5.4(2.4)$ \\
m-SaRa & $9.7(3.3)$ & $16.0(4.3)$ & $22.3(4.7)$ & $23.2(4.7)$ & $21.8(4.7)$ & $21.9(4.9)$ \\
Multiple-sample CBS & $17.4(5.6)$ & $17.7(5.5)$ & $3.9(2.2)$ & $4.5(2.3)$ & $11.2(3.7)$ & $38.1(7.0)$ \\
Multiple-sample m-SaRa & $4.0(2.1)$ & $1.6(1.4)$ & $2.0(1.5)$ & $2.0(1.5)$ & $0.0(0.1)$ & $0.0(0.1)$ \\
\hline
\end{tabular}


Supplement Table 4. True and false positives grouped by the changepoints (CP1-CP6) for the simulation with dependent errors in Supplement Section 2, Standard errors are shown in parentheses.

(a) Average number of true positives.

\begin{tabular}{ccccccc}
\hline \hline & CP1 & CP2 & CP3 & CP4 & CP5 & CP6 \\
Number of Carriers & 20 & 20 & 50 & 50 & 100 & 100 \\
\hline fast CBS & $19.8(0.4)$ & $19.9(0.3)$ & $47.4(1.6)$ & $47.5(1.5)$ & $93.9(2.4)$ & $93.9(2.4)$ \\
CBS-SS & $19.8(0.4)$ & $19.9(0.3)$ & $47.4(1.6)$ & $47.4(1.5)$ & $93.8(2.4)$ & $93.9(2.4)$ \\
m-SaRa & $19.8(0.4)$ & $19.8(0.4)$ & $47.2(1.6)$ & $47.4(1.5)$ & $91.7(2.7)$ & $91.8(2.8)$ \\
Multiple-sample CBS & $20.0(0.0)$ & $20.0(0.0)$ & $50.0(0.2)$ & $50.0(0.2)$ & $100.0(0.0)$ & $100.0(0.0)$ \\
Multiple-sample m-SaRa & $20.0(0.0)$ & $20.0(0.0)$ & $50.0(0.2)$ & $50.0(0.2)$ & $100.0(0.0)$ & $100.0(0.0)$ \\
\hline
\end{tabular}

(b) Average number of false positives.

\begin{tabular}{ccccccc}
\hline \hline & CP1 & CP2 & CP3 & CP4 & CP5 & CP6 \\
\hline fast CBS & $1.1(1.0)$ & $1.2(1.1)$ & $1.2(1.1)$ & $1.2(1.1)$ & $1.1(1.1)$ & $1.1(1.1)$ \\
CBS-SS & $0.5(0.7)$ & $0.6(0.8)$ & $0.8(0.9)$ & $0.9(0.9)$ & $0.9(1.0)$ & $0.9(0.9)$ \\
m-SaRa & $4.9(2.2)$ & $6.8(2.5)$ & $8.3(3.0)$ & $8.6(2.8)$ & $8.6(2.9)$ & $8.6(2.9)$ \\
Multiple-sample CBS & $3.7(1.9)$ & $3.8(1.9)$ & $2.2(1.5)$ & $2.4(1.5)$ & $2.3(1.7)$ & $2.2(1.9)$ \\
Multiple-sample m-SaRa & $1.2(1.1)$ & $0.4(0.7)$ & $0.8(0.8)$ & $0.8(0.8)$ & $0.0(0.1)$ & $0.0(0.0)$ \\
\hline
\end{tabular}


Supplement Table 5. True and false positives grouped by the changepoints (CP1-CP6) for the simulation with GC content related trend in Section 4.2.2. Standard errors are shown in parentheses.

(a) Average number of true positives.

\begin{tabular}{ccccccc}
\hline \hline & CP1 & CP2 & CP3 & CP4 & CP5 & CP6 \\
Number of Carriers & 20 & 20 & 50 & 50 & 100 & 100 \\
\hline fast CBS & $19.9(0.4)$ & $19.9(0.4)$ & $47.0(1.6)$ & $47.0(1.7)$ & $93.3(2.7)$ & $93.2(2.9)$ \\
CBS-SS & $19.9(0.4)$ & $19.9(0.4)$ & $46.9(1.6)$ & $47.0(1.7)$ & $93.2(2.8)$ & $93.1(2.9)$ \\
m-SaRa & $19.8(0.4)$ & $19.8(0.4)$ & $47.2(1.6)$ & $47.1(1.6)$ & $91.6(2.7)$ & $91.5(2.8)$ \\
Multiple-sample CBS & $19.1(4.1)$ & $19.0(4.4)$ & $43.6(15.8)$ & $45.3(14.2)$ & $100.0(0.0)$ & $100.0(0.0)$ \\
Multiple-sample m-SaRa & $20.0(0.0)$ & $20.0(0.0)$ & $49.9(0.3)$ & $49.9(0.3)$ & $100.0(0.0)$ & $100.0(0.0)$ \\
\hline
\end{tabular}

(b) Average number of false positives.

\begin{tabular}{ccccccc}
\hline \hline & CP1 & CP2 & CP3 & CP4 & CP5 & CP6 \\
\hline fast CBS & $3.5(3.2)$ & $4.2(3.5)$ & $3.8(2.9)$ & $3.9(2.9)$ & $3.2(2.3)$ & $3.4(2.7)$ \\
CBS-SS & $1.0(1.4)$ & $2.2(2.2)$ & $2.9(2.2)$ & $3.0(2.4)$ & $2.6(2.1)$ & $2.8(2.3)$ \\
m-SaRa & $6.3(3.6)$ & $10.2(5.4)$ & $13.8(5.9)$ & $14.0(6.0)$ & $13.1(5.3)$ & $13.5(5.7)$ \\
Multiple-sample CBS & $6.4(5.4)$ & $11.9(13.3)$ & $11.4(20.3)$ & $8.1(8.3)$ & $33.0(34.5)$ & $31.6(30.9)$ \\
Multiple-sample m-SaRa & $2.1(2.3)$ & $0.7(1.1)$ & $1.3(1.7)$ & $1.2(1.7)$ & $0.0(0.0)$ & $0.0(0.2)$ \\
\hline
\end{tabular}


Supplement Table 6 . True and false positives grouped by the changepoints (CP1-CP6) for the simulation with change-points that are slightly different in each carrier in Section 4.2.3. Standard errors are shown in parentheses.

(a) Average number of true positives.

\begin{tabular}{ccccccc}
\hline \hline & CP1 & CP2 & CP3 & CP4 & CP5 & CP6 \\
Number of Carriers & 20 & 20 & 50 & 50 & 100 & 100 \\
\hline fast CBS & $19.6(0.6)$ & $19.6(0.7)$ & $46.2(1.9)$ & $46.1(1.9)$ & $91.4(2.8)$ & $91.5(2.7)$ \\
CBS-SS & $19.6(0.6)$ & $19.6(0.7)$ & $46.2(1.9)$ & $46.1(1.9)$ & $91.4(2.8)$ & $91.5(2.7)$ \\
m-SaRa & $19.5(0.7)$ & $19.5(0.7)$ & $45.9(2.0)$ & $45.9(1.9)$ & $88.7(3.2)$ & $88.9(3.2)$ \\
Multiple-sample CBS & $20.0(0.0)$ & $20.0(0.0)$ & $49.8(0.5)$ & $49.9(0.3)$ & $100.0(0.0)$ & $100.0(0.0)$ \\
Multiple-sample m-SaRa & $20.0(0.0)$ & $20.0(0.0)$ & $49.7(0.6)$ & $49.7(0.6)$ & $100.0(0.0)$ & $100.0(0.0)$ \\
\hline
\end{tabular}

(b) Average number of false positives.

\begin{tabular}{ccccccc}
\hline \hline & CP1 & CP2 & CP3 & CP4 & CP5 & CP6 \\
\hline fast CBS & $0.3(0.6)$ & $0.3(0.6)$ & $0.3(0.5)$ & $0.3(0.5)$ & $0.2(0.5)$ & $0.2(0.5)$ \\
CBS-SS & $0.2(0.4)$ & $0.2(0.4)$ & $0.2(0.5)$ & $0.2(0.5)$ & $0.2(0.5)$ & $0.2(0.5)$ \\
m-SaRa & $3.0(1.8)$ & $4.0(2.0)$ & $4.7(2.2)$ & $5.1(2.2)$ & $4.9(2.2)$ & $5.0(2.2)$ \\
Multiple-sample CBS & $2.7(1.7)$ & $2.3(1.5)$ & $2.9(1.7)$ & $2.8(1.7)$ & $1.3(1.1)$ & $1.1(1.0)$ \\
Multiple-sample m-SaRa & $0.3(0.5)$ & $0.1(0.3)$ & $1.0(1.3)$ & $1.0(1.3)$ & $0.0(0.0)$ & $0.0(0.0)$ \\
\hline
\end{tabular}

